\title{
Factors associated with emergent colectomy in patients with neutropenic enterocolitis
}

\author{
Omar Vergara-Fernández ${ }^{1} \cdot$ Mario Trejo-Avila ${ }^{1}$ (1) $\cdot$ Danilo Solórzano-Vicuña $^{1} \cdot$ Oscar Santes $^{1} \cdot$ Noel Salgado-Nesme $^{1}$
}

Received: 6 February 2019 / Accepted: 25 March 2019 / Published online: 5 April 2019

(C) Springer-Verlag GmbH Germany, part of Springer Nature 2019

\begin{abstract}
Purpose Neutropenic enterocolitis (NEC) is a severe complication of neutropenia. NEC is characterized by segmental ulceration, intramural inflammation, and necrosis. Factors present in patients who underwent colectomy have never been studied. The present study aimed to describe the clinical factors present in patients who underwent emergent colectomy for the treatment of neutropenic enterocolitis.

Methods Patients admitted with neutropenic enterocolitis from November 2009 to May 2018 were retrospectively analyzed. Logistic regression analysis was used to determine clinical factors associated with emergent colectomy.

Results Thirty-nine patients with NEC were identified. All patients had a hematological disorder. Medical treatment was the only management in 30 (76.9\%) patients, and $9(23.1 \%)$ patients underwent colectomy. No differences were found between the treatment groups regarding sex, age, or comorbidities. Patients were more likely to undergo colectomy if they developed abdominal distention $(\mathrm{OR}=12, p=0.027)$, hemodynamic failure $(\mathrm{OR}=6, p=0.042)$, respiratory failure $(\mathrm{OR}=17.5, p=$ $0.002)$, multi-organic failure ( $\mathrm{OR}=9.6, p=0.012)$, and if they required ICU admission ( $\mathrm{OR}=11.5, p=0.007)$. Respiratory failure was the only independent risk factor for colectomy in multivariable analysis. In-hospital mortality for the medical and surgical treatment groups was $13.3 \%(n=4)$ and $44.4 \%(n=4)$, respectively $(p=0.043)$.

Conclusions In our study, most NEC patients were treated conservatively. Patients were more likely to undergo colectomy if they developed organ failures or required ICU admission. Early surgical consultation is suggested in all patients with NEC.
\end{abstract}

Keywords Neutropenic enterocolitis $\cdot$ Typhlitis $\cdot$ Febrile neutropenia $\cdot$ Colectomy

\section{Introduction}

Despite recent advances in prevention, febrile neutropenia remains a chemotherapy complication for patients with cancer [1]. Formerly referred to as typhlitis (from the Greek typhlon, or cecum), neutropenic enterocolitis (NEC) is a serious complication of neutropenia. It is characterized by segmental ulceration and intramural inflammation with necrosis of the gut (especially in the ileal-cecum-ascending colon area) $[2,3]$. NEC was first described in the 1970s as a complication of the treatment of childhood leukemias [4-6]. It occurs most frequently after intensive chemotherapy in acute leukemias

Mario Trejo-Avila

mario.trejo.avila@gmail.com

1 Department of Colorectal Surgery, Instituto Nacional de Ciencias Médicas y Nutrición "Salvador Zubirán”, Vasco de Quiroga, 15, Sección XVI, Tlalpan, 14080 Mexico City, Mexico
[7]. The actual incidence of NEC is unknown, and reports range from 0.8 to $26 \%[2,3,7]$. NEC is considered as the most common gastrointestinal infection related to neutropenia [8].

The precise pathogenesis of NEC is undefined [8]. The proposed pathogenesis is immunosuppression secondary to neutropenia, in conjunction with chemotherapy cytotoxicity, tumor infiltration, and inflammatory reaction, which lead to direct mucosal injury, loss of gut barrier function, and microbial translocation $[2,3]$. Following the microbial invasion, production and release of bacterial endotoxins result in inflammation, edema, ulceration, transmural necrosis, perforation, and septic shock [8].

A recent meta-analysis estimated that the overall mortality of NEC was $42.2 \%$ (95\% CI $=40.2-44.2)$, and surgery was not associated with an elevated risk of mortality compared to medical treatment [2]. Generally, if conservative antibiotic treatment is unsuccessful, the indication for surgery and resection of the necrotic tissue regions must be performed early, 
even before regeneration of the bone marrow [9]. Other indications for surgery include bowel perforation, massive gastrointestinal bleeding, or whenever a concomitant problem that requires surgery is present $[2,10]$. Surgical treatment outcomes have never been evaluated against conservative treatment, the indications for surgical treatment are ambiguous, the predictors of treatment failure or need for surgery are lacking, and the surgical literature about this topic is scarce [2]. Further, publications concerning the optimal timing for surgical therapy, patients at risk, and prognosis after surgery are unavailable. Therefore, the evidence for recommendations regarding the optimal management of NEC is mostly expert opinion [10].

The present study aimed to describe the clinical factors present in patients who underwent emergent colectomy for the treatment of neutropenic enterocolitis.

\section{Materials and methods}

Patients were retrospectively identified and analyzed using the hospital registry. All adult patients ( $\geq 18$ years old) admitted at the Instituto Nacional de Ciencias Médicas y Nutrición Salvador Zubirán in Mexico City, Mexico, with the diagnosis of neutropenic enterocolitis (NEC) between November 2009 and May 2018 were included. Patients with incomplete data were excluded from the analysis. This study was approved by the local Institutional Review Board.

Neutropenia was defined as an absolute neutrophil count (ANC) of $<1000$ cells/ $\mu \mathrm{L}[1]$. Patients were classified according to the definition of severe neutropenia (ANC $<500$ cells/ $\mu \mathrm{L}$ or an ANC that was expected to decrease below 500 cells/ $\mu \mathrm{L}$ in the next $48 \mathrm{~h}$ ) and profound neutropenia (ANC $<100$ cells $/ \mu \mathrm{L})[1,11]$. Febrile neutropenia was defined as single temperature measurement by oral or tympanic membrane of $\geq 38.3^{\circ} \mathrm{C}\left(101^{\circ} \mathrm{F}\right)$ or a temperature of $>38.0^{\circ} \mathrm{C}\left(100.4^{\circ} \mathrm{F}\right)$ for more than $1 \mathrm{~h}[1,11]$. Neutropenic enterocolitis was defined as the presence of febrile neutropenia with abdominal pain (at least 3, determined by the patient using a visual analogous scale (VAS) pain score ranging from 1 to 10 ), and bowel wall thickening ( $>4 \mathrm{~mm}$ on transversal scan over more than $30 \mathrm{~mm}$ in longitudinal scan) in any segment evident on computed tomography images [7]. All patients were evaluated according to the Multinational Association for Supportive Care in Cancer (MASCC) Febrile Neutropenia Risk Stratification Index. Included patients had a MASCC score $<21$ [12].

The clinical variables including age $(<50$ and $\geq 50$ years old), sex, and the underlying hematological malignancy were registered. Comorbidity was assessed using the Charlson comorbidity index (CCI) [13]. Patients included received chemotherapy within the previous 6 weeks or had a known bone marrow failure syndrome with neutropenia [1]. Symptomatic and physical findings that included diarrhea ( $>3$ bowel movements/day), acute abdominal pain (VAS $>3$ ), nausea or vomiting, acute lower gastrointestinal bleeding (hematochezia, or rectal bleeding), fever (temperature $\geq$ $38{ }^{\circ} \mathrm{C}$ ), abdominal distention (determined by surgeon during physical examination), and peritoneal signs (guarding and rebound tenderness) were recorded.

Laboratory tests at presentation to the emergency department (complete blood count, $\mathrm{ANC}$, and albumin) were registered. Blood cultures at admission $(<72 \mathrm{~h})$ and stool testing (including Clostridium difficile toxin) were evaluated. All patients had a CT scan. The affected colonic segment (right colon, left colon, or pan-colonic) and the presence of free intraperitoneal air were determined and registered in the database.

Severity of the disease was established taking into account the following parameters: admission to ICU (intensive care unit), hemodynamic/cardiovascular failure (persistent systolic hypotension $\leq 100 \mathrm{mmHg}$ or mean arterial pressure < $70 \mathrm{mmHg}$ ), respiratory failure (tachypnea $\geq 22 / \mathrm{min}$ or $\mathrm{PaO} 2 / \mathrm{FiO} 2<400$ ), renal failure (creatinine $>1.2$, or urine output of $<500 \mathrm{~mL} /$ day), altered mentation (Glasgow Coma Scale < 14), liver failure (total bilirubin $>1.2 \mathrm{mg} / \mathrm{dL}$ ), multiorganic failure ( $\geq 3$ systemic failures for $\geq 72 \mathrm{~h}$ ), and treatment with mechanical ventilation and vasopressors (for at least $1 \mathrm{~h}$ ) [14]. In-hospital mortality attributed to neutropenic enterocolitis or its complications was analyzed. The cause of inhospital mortality was registered.

Patients were categorized into two groups depending on the treatment required. The medical treatment group included patients who did not undergo surgery for NEC or its complications. The surgical treatment group comprised patients who underwent emergent colectomy for the treatment of NEC or its complications. Variables were dichotomized and compared between these two groups.

All patients were assessed by a multidisciplinary team formed by internists, hematologists, oncologists, infectious disease specialists, critical care providers, and colorectal surgeons. All patients received medical treatment according to clinical practice guidelines; it involved general supportive care, bowel rest (with parenteral nutrition as needed), and broad-spectrum antibiotics $[1,11,15]$. Antiviral and antifungal drug administration was determined according to each patient risk $[1,11]$. All surgical procedures were considered emergency operations, all surgeries were approached by laparotomy, and were supervised or performed by a certified colorectal surgeon. Surgical variables included the type of procedure (segmental or total abdominal colectomy), postoperative morbidity, and in-hospital mortality.

\section{Statistical analysis}

Categorical data were presented as totals $(n)$, proportions as percentages $(\%)$, and continuous variables were expressed as 
means including standard deviations $( \pm \mathrm{SD})$. Categorical data were compared using the Pearson's Chi-square test or Fisher's exact test. Continuous variables were compared with the Student's $t$ test. All tests were two-sided and used an alpha of 0.05 .

Patients who underwent surgical therapy were compared with those who required only medical treatment. Univariate binomial logistic regression analysis was performed. Variables with a $p$ value inferior to 0.10 in the univariate analysis were considered as potential risk factors (predictors of colectomy) and were entered into the multivariate backward logistic regression analysis. The variables representing the lowest risk were considered to be the reference group ( $\mathrm{OR}=1)$. Odds ratios and $95 \%$ confidence intervals $(95 \% \mathrm{CI})$ were calculated.

All $p$ values were two-tailed, and a $p$ value $<0.05$ was considered to be statistically significant. The analysis was performed employing SPSS Version 22.0 (IBM Corporation, Armonk, New York, NY).

\section{Results}

Our selection criteria yielded 39 cases of NEC. Emergent surgery was conducted in $9(23.1 \%)$ patients, and 30 $(76.9 \%)$ patients received medical treatment exclusively. No differences were found regarding sex, age, and CCI among the groups (Table 1). All patients had a hematological disorder. Acute lymphoblastic leukemia was the most frequent diagnosis (medical group $43.3 \%$ vs. surgical group $33.3 \%, p=$ $0.711)$.

Aside from fever and abdominal pain, the frequency of symptoms and signs at presentation are shown in Table 1. Clinical variables that differed significantly between medical and surgical groups were the presence of peritoneal signs on physical examination ( 0 vs. $55.6 \%, p<0.001$ ), abdominal distention (40 vs. $88.9 \%, p=0.010$ ) and free intraperitoneal air on CT scan ( 0 vs. $33.3 \%, p=0.001$ ). Laboratory test results at admission were similar between the groups (Table 1). The mean ANC (cells $/ \mu \mathrm{L})$ was $145.1( \pm 136)$ in the medical group and $117.7( \pm 88.4)$ in the surgical group $(p=0.575)$. There was a preponderance of profound neutropenia in both groups. Blood cultures were positive in $13(43.3 \%)$ patients of the medical group and $4(44.4 \%)$ patients of the surgical group. The most common isolate was Escherichia coli. Fifteen concomitant infections were identified in 11 patients in the medical group, including pneumonia $(n=6)$, acute sinusitis $(n=$ $3)$, oral cavity infections $(n=3)$, skin infections $(n=2)$, and splenic abscess $(n=1)$. In the surgical group, there were four concurrent infections in four patients, including pneumonia $(n=3)$ and skin infection $(n=1)$.

A higher proportion of patients in the surgical group required admission to the ICU. Those patients experienced a significantly higher rate of preoperative adverse events including shock (hemodynamic failure), respiratory failure, and multi-organic failure (Table 2).

On univariate analysis (Table 3), patients were more likely to undergo colectomy if they developed abdominal distention $(\mathrm{OR}=12, p=0.027)$, hemodynamic failure or shock $(\mathrm{OR}=6$, $p=0.042)$, respiratory failure $(\mathrm{OR}=17.5, p=0.002)$, multiorganic failure $(\mathrm{OR}=9.6, p=0.012)$, if they required vasopressors $(\mathrm{OR}=12, \mathrm{p}=0.027)$ or mechanical ventilation $(\mathrm{OR}=17.5, p=0.002)$, and if they required ICU admission $(\mathrm{OR}=11.5, p=0.007)$. On multivariate analysis, the only preoperative factor that was associated significantly with emergent colectomy was the presence of respiratory failure and mechanical ventilation requirement $(\mathrm{OR}=17.5,95 \% \mathrm{CI}$ : 2.76-110.37, $p=0.002$ ).

The procedures performed in the surgical group were segmental colectomy $(n=6,66.7 \%)$ and total abdominal colectomy $(n=3,33.3 \%)$. Terminal ostomy was created in all patients. Postoperative complications included minor wound complications $(n=2)$, prolonged ileus $(n=2)$, and septic shock $(n=2)$. Preoperatively, seven $(77.7 \%)$ patients were admitted to the ICU. Postoperatively, eight (88.8\%) patients required admission to the ICU.

The overall inpatient mortality rate was $20.5 \%$. The mortality after colectomy was $44.4 \%(n=4)$ and the mortality after medical treatment was $13.3 \%(n=4)$. The cause of death after colectomy was abdominal septic shock with multiorganic failure in all patients $(n=4)$. The cause of death in the medical treatment group was ventilator-associated pneumonia $(n=3)$, and abdominal septic shock $(n=1)$.

\section{Discussion}

In this retrospective study, patients were more likely to undergo colectomy if they developed abdominal distention, hemodynamic failure, respiratory failure, multi-organic failure, and if they required ICU admission. We observed that the mortality rate after colectomy was $44.4 \%$. Based on these results, we believe that early surgery before the development of shock or organ failure could decrease the mortality rates after emergent colectomy.

Patients with febrile neutropenia should be treated as an emergency, requiring immediate medical attention. NEC is a potentially lethal complication of anti-neoplastic chemotherapy and has been reported in association with solid, hematologic, and lymphoproliferative malignant tumors [8]. Other conditions linked to the development of NEC are aplastic anemia, cyclic neutropenia, use of immunosuppressive therapies, and acquired immunodeficiency syndrome [8].

Gorschluter et al. proposed the most widely accepted definition of NEC [7]. It includes the presence of fever, abdominal pain, and bowel wall thickening on US or CT scan. 
Table 1 Comparison of demographics and comorbidities of patients admitted with neutropenic enterocolitis

\begin{tabular}{|c|c|c|c|}
\hline & $\begin{array}{l}\text { Medical treatment group } \\
(n=30)\end{array}$ & $\begin{array}{l}\text { Surgical treatment group } \\
(n=9)\end{array}$ & $P<0.05$ \\
\hline Sex & & & 0.292 \\
\hline Female & $14(46.7)$ & $6(66.7)$ & \\
\hline Male & $16(53.5)$ & $3(33.3)$ & \\
\hline Age, $n(\%)$ & & & 0.107 \\
\hline$<50$ years & $22(73.3)$ & $4(44.4)$ & \\
\hline$\geq 50$ years & $8(26.7)$ & $5(55.6)$ & \\
\hline \multicolumn{4}{|l|}{ Hematological diagnosis, $n(\%)$} \\
\hline Acute lymphoblastic leukemia & $13(43.3)$ & $3(33.3)$ & 0.711 \\
\hline Acute myeloid leukemia & $5(16.6)$ & $3(33.3)$ & 0.354 \\
\hline Aplastic anemia & $1(3.3)$ & 0 & 1.000 \\
\hline Lymphomas & $8(26.6)$ & $1(11.1)$ & 0.654 \\
\hline Chronic myeloid leukemia & 0 & $2(22.2)$ & 0.048 \\
\hline Myelodysplastic syndrome & $1(3.3)$ & 0 & 1.000 \\
\hline Cyclic neutropenia & $1(3.3)$ & 0 & 1.000 \\
\hline Multiple myeloma & $1(3.3)$ & 0 & 1.000 \\
\hline Charlson comorbidity index, $n(\%)$ & & & 0.884 \\
\hline 2 & $24(80)$ & $7(77.8)$ & \\
\hline$\geq 3$ & $6(20)$ & $2(22.2)$ & \\
\hline Diarrhea, $n(\%)$ & & & 0.907 \\
\hline No & $14(46.7)$ & $4(44.4)$ & \\
\hline Yes & $16(53.3)$ & $5(55.6)$ & \\
\hline Nausea/vomiting, $n(\%)$ & & & 0.674 \\
\hline No & $19(63.3)$ & $5(55.6)$ & \\
\hline Yes & $11(36.7)$ & $4(44.4)$ & \\
\hline Low GI bleeding, $n(\%)$ & & & 0.043 \\
\hline No & $26(86.7)$ & $5(55.6)$ & \\
\hline Yes & $4(13.3)$ & $4(44.4)$ & \\
\hline Abdominal distention, $n(\%)$ & & & 0.010 \\
\hline No & $18(60)$ & $1(11.1)$ & \\
\hline Yes & $12(40)$ & $8(88.9)$ & \\
\hline Peritonitis, $n(\%)$ & & & $<0.001$ \\
\hline No & $30(100)$ & $4(44.4)$ & \\
\hline Yes & 0 & $5(55.6)$ & \\
\hline $\begin{array}{l}\text { Absolute neutrophil count (cells } / \mu \mathrm{L}) \\
\text { mean }(\mathrm{SD})\end{array}$ & $145.1(136)$ & $117.7(88.4)$ & 0.575 \\
\hline Neutropenia, $n(\%)$ & & & 0.907 \\
\hline Severe & $14(46.7)$ & $4(44.4)$ & \\
\hline Profound & $16(53.3)$ & $5(55.6)$ & \\
\hline Treatment with GCS-F, $n(\%)$ & & & 0.277 \\
\hline Yes & $25(83.3)$ & $6(66.7)$ & \\
\hline No & $5(16.7)$ & $3(33.3)$ & \\
\hline \multicolumn{4}{|l|}{ Laboratory values at admission } \\
\hline WBC, mean (SD) & $0.72(0.7)$ & $0.41(0.6)$ & 0.243 \\
\hline Hemoglobin, mean (SD) & $8.94(2.6)$ & $8.17(1.5)$ & 0.415 \\
\hline Platelets, mean (SD) & $107.3(157.4)$ & $33.0(54.5)$ & 0.176 \\
\hline Albumin $<3 \mathrm{~g} / \mathrm{dL}, n(\%)$ & $17(56.6)$ & $7(77.8)$ & 0.253 \\
\hline Affected colonic segment, $n(\%)$ & & & 0.972 \\
\hline Right colon & $18(60)$ & $5(55.6)$ & \\
\hline Left colon & $3(10)$ & $1(11.1)$ & \\
\hline Pancolonic & $9(30)$ & $3(33.3)$ & \\
\hline Bowel wall thickening (mm), mean & $5.16(0.98)$ & $5.77(1.09)$ & 0.120 \\
\hline
\end{tabular}


Table 1 (continued)

\begin{tabular}{|c|c|c|c|}
\hline & $\begin{array}{l}\text { Medical treatment group } \\
(n=30)\end{array}$ & $\begin{array}{l}\text { Surgical treatment group } \\
(n=9)\end{array}$ & $P<0.05$ \\
\hline Free intraperitoneal air, $n(\%)$ & & & 0.001 \\
\hline No & $30(100)$ & $6(66.7)$ & \\
\hline Yes & 0 & $3(33.3)$ & \\
\hline Bacteremia/fungemia & & & 0.952 \\
\hline No & $17(56.7)$ & $5(55.6)$ & \\
\hline Yes & $13(43.3)$ & $4(44.4)$ & \\
\hline \multicolumn{4}{|c|}{ Microbiological isolations from blood cultures, $n(\%)$} \\
\hline Negative & $17(56.7)$ & $5(55.6)$ & 1.000 \\
\hline Escherichia coli & $8(26.6)$ & $2(22.2)$ & 1.000 \\
\hline ESBL-Escherichia coli & $2(6.6)$ & $1(11.1)$ & 0.555 \\
\hline Klebsiella pneumoniae & $1(3.3)$ & $1(11.1)$ & 0.413 \\
\hline Proteus mirabilis & $1(3.3)$ & 0 & 1.000 \\
\hline Candida sp. & $1(3.3)$ & 0 & 1.000 \\
\hline \multicolumn{4}{|c|}{ Microbiological isolations from stool, $n(\%)$} \\
\hline Negative & $24(80)$ & $7(77.8)$ & 1.000 \\
\hline Clostridium difficile & $2(6.6)$ & $1(11.1)$ & 0.660 \\
\hline Campylobacter & $1(3.3)$ & 0 & 1.000 \\
\hline Cryptosporidium & $1(3.3)$ & 0 & 1.000 \\
\hline Other infections, $n(\%)$ & & & 0.674 \\
\hline No & $19(63.3)$ & $5(55.6)$ & \\
\hline Yes & $11(36.7)$ & $4(44.4)$ & \\
\hline Pneumonia & 6 & 3 & \\
\hline Acute sinusitis & 3 & 0 & \\
\hline Skin & 2 & 1 & \\
\hline Oral cavity & 3 & 0 & \\
\hline Splenic abscess & 1 & 0 & \\
\hline
\end{tabular}

GCSF granulocyte colony-stimulating factor, GI gastro intestinal, WBC white blood cells, ESBL extendedspectrum beta-lactamase

Histologic examination remains the gold standard in NEC; however, it is not helpful for clinical decision-making [3, 7].

The suggested initial workup for patients with suspected NEC includes blood cultures (aerobes, anaerobes, and fungi), C. difficile toxin assay, and abdominal CT scan $[2,10]$. CT findings compatible with NEC include diffuse bowel wall thickening, intramural edema or hemorrhage, paracolonic fluid, inflammatory mass, free intraperitoneal air, and pneumatosis intestinalis $[8,16]$. Cartoni et al. [17] reported a mortality rate of $60 \%$ due to NEC in patients with a colonic wall thickness of $10 \mathrm{~mm}$, opposed to a mortality rate of $4.2 \%$ in patients with a mural thickening less than $10 \mathrm{~mm}$.

Initial medical treatment consists of bowel rest, fluid resuscitation, total parenteral nutrition, and broad-spectrum antibiotic therapy. Consideration for antifungal therapy is reasonable, although published guidelines did not provide a recommendation for its routine use [11]. Correction of thrombocytopenia and clotting abnormalities is necessary especially in patients considered for surgery [16]. Granulocyte colonystimulating factor (G-CSF) should be considered in patients with fever and neutropenia who are at high risk for infectionassociated complications, or who have prognostic factors that are predictive of poor clinical outcomes [18].

NEC represents a spectrum of disease ranging from mild, self-limited bowel inflammation to fulminant necrosis and perforation [19]. Shamberger et al. [20] proposed criteria for surgical interventions in NEC, including persistent gastrointestinal bleeding, free intraperitoneal air indicative of bowel perforation, clinical deterioration despite optimal medical treatment, and the presence of other indications for surgery like appendicitis and cholecystitis. Other potential complications reported are an intestinal fistula, pseudo-obstruction, ileus, and intra-abdominal abscess formation [6-8, 19-22].

The ileocecum is the most vulnerable region to NEC, based on abundant lymphoid tissue, increased stasis and ability to distend, and decreased vascularity [3]. We confirmed this finding in our study, in which exclusive affectation of the right colon (ileocecal area) was present in $60 \%$ of the medical group patients and $55.6 \%$ of the surgical group patients. Pancolonic 
Table 2 Comparison of acute care variables of patients with neutropenic enterocolitis that underwent medical versus surgical treatment

\begin{tabular}{|c|c|c|c|}
\hline & $\begin{array}{l}\text { Medical treatment } \\
\text { group }(n=30)\end{array}$ & $\begin{array}{l}\text { Surgical treatment } \\
\text { group }(n=9)\end{array}$ & $P<0.05$ \\
\hline Critical care/ICU admission, $n(\%)$ & & & 0.003 \\
\hline No & $23(76.7)$ & $2(22.2)$ & \\
\hline Yes & $7(23.3)$ & $7(77.8)$ & \\
\hline Hemodynamic/CV failure, $n(\%)$ & & & 0.030 \\
\hline No & $19(63.3)$ & $2(22.2)$ & \\
\hline Yes & $11(36.7)$ & $7(77.8)$ & \\
\hline Altered mental status, $n(\%)$ & & & 0.177 \\
\hline No & $28(93.3)$ & $7(77.8)$ & \\
\hline Yes & $2(6.7)$ & $2(22.2)$ & \\
\hline Respiratory failure, $n(\%)$ & & & $<0.001$ \\
\hline No & $25(83.3)$ & $2(22.2)$ & \\
\hline Yes & $5(16.7)$ & $7(77.8)$ & \\
\hline Renal failure, $n(\%)$ & & & 0.703 \\
\hline No & $24(80)$ & $6(66.7)$ & \\
\hline Yes & $6(20)$ & $3(33.3)$ & \\
\hline Liver failure, $n(\%)$ & & & 0.354 \\
\hline No & $29(96.7)$ & $8(88.9)$ & \\
\hline Yes & $1(3.3)$ & $1(11.1)$ & \\
\hline Multiorganic failure, $n(\%)$ & & & 0.006 \\
\hline No & $22(73.3)$ & $2(22.2)$ & \\
\hline Yes & $8(26.7)$ & $7(77.8)$ & \\
\hline Mechanical ventilation, $n(\%)$ & & & $<0.001$ \\
\hline No & $25(83.3)$ & $2(22.2)$ & \\
\hline Yes & $5(16.7)$ & $7(77.8)$ & \\
\hline Vasopressors, $n(\%)$ & & & 0.010 \\
\hline No & $18(60)$ & $1(11.1)$ & \\
\hline Yes & $12(40)$ & $8(88.9)$ & \\
\hline In-hospital mortality & & & 0.043 \\
\hline No & $26(86.7)$ & $5(55.6)$ & \\
\hline Yes & $4(13.3)$ & $4(44.4)$ & \\
\hline
\end{tabular}

$I C U$ intensive care unit, $C V$ cardiovascular involvement was observed in $30 \%$ and $33.3 \%$ of patients in the medical and surgical groups, respectively. This anatomical fact is important in order to have a preoperative surgical plan.

As reflected in the univariate analysis, patients who underwent surgery had at least one organic failure, and the majority of patients required admission to the ICU before surgery. We assume that it is the reflection of a delay in the decision to proceed to surgical therapy. Based on the severity of NEC in patients who needed surgery, early surgical consultation and identification of patients at risk of developing multiorganic failures should be pursued.

A subtle difference in the proportion of in-hospital mortality was found. However, we cannot draw conclusions because we were not evaluating effectiveness between the two treatment modalities neither we were evaluating survival. In a recent meta-analysis, abdominal surgery was not deleterious and was associated with a favorable outcome, compared to conservative medical treatment, with an OR of $0.41(95 \%$ $\mathrm{CI}=0.23-0.74 ; p=0.003)$ [2].
Physicians are usually reluctant to select surgery as the first-choice treatment [2], but we believe that leaving surgery as a last resort could be detrimental to the patients. Saillard et al. [2] found that surgery for NEC was not deleterious, regardless of underlying malignancy, lapse time, and the presence of neutropenia at the time of surgery. These findings suggest that surgery should not be delayed until resolution of neutropenia. However, we should be aware of several limitations and risk of bias inherent to the heterogeneity of studies included in this systematic review and meta-analysis. In our opinion, several clinical questions regarding the surgical treatment of neutropenic enterocolitis remain to be answered. Some of these questions are the definition of medical treatment failure, identification of patients at risk of developing neutropenic enterocolitis, and risk of recurrence.

The standard operative approach is an exploratory laparotomy with resection of the involved bowel and creation of an ostomy [6-8, 19-26]. Primary anastomosis in cases of a severely immunocompromised host with septic shock is not 
Table 3 Unadjusted logistic regression analysis of factors associated with the need for surgical treatment in hospitalized patients with neutropenic enterocolitis

\begin{tabular}{|c|c|c|}
\hline Predictive factors for colectomy & $\begin{array}{l}\text { Unadjusted OR } \\
(95 \% \mathrm{CI})\end{array}$ & $P$ value \\
\hline Abdominal distention, $n(\%)$ & & 0.027 \\
\hline No & 1.0 & \\
\hline Yes & $12(1.32-108.67)$ & \\
\hline Low GI bleeding, $n(\%)$ & & 0.055 \\
\hline No & 1.0 & \\
\hline Yes & $5.2(0.96-28.02)$ & \\
\hline Critical care/ICU admission, $n(\%)$ & & 0.007 \\
\hline No & 1.0 & \\
\hline Yes & $11.5(1.93-68.51)$ & \\
\hline Hemodynamic/CV failure, $n(\%)$ & & 0.042 \\
\hline 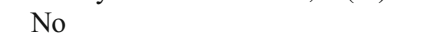 & 1.0 & \\
\hline Yes & $6(1.06-34.37)$ & \\
\hline Respiratory failure, $n(\%)$ & & 0.002 \\
\hline No & 1.0 & \\
\hline Yes & $17.5(2.78-110.37)$ & \\
\hline Multiorganic failure, $n(\%)$ & & 0.012 \\
\hline No & 1.0 & \\
\hline Yes & $9.6(1.64-56.37)$ & \\
\hline Mechanical ventilation, $n(\%)$ & & 0.002 \\
\hline No & 1.0 & \\
\hline Yes & $17.5(2.76-110.37)$ & \\
\hline Vasopressors, $n(\%)$ & & 0.027 \\
\hline No & 1.0 & \\
\hline Yes & $12.0(1.33-108.67)$ & \\
\hline
\end{tabular}

The reference category has an odds ratio of 1.00

GI gastrointestinal, $I C U$ intensive care unit, $C V$ cardiovascular

advised; however, there are no studies about it [16, 19]. Ileostomy for fecal diversion in pan-colonic severe disease has been suggested, but again, there are no studies to support this [19]. Drainage of the necrotic region without resection seems to be insufficient $[16,19,25]$. The role of laparoscopic surgery in this group of patients has not been evaluated [8].

Our study has several limitations. The main limitations are that our study has an observational retrospective design, the sample size is small, and our data derived from a single institution. Although a multivariate logistic regression analysis was performed, the sample size was considered too small for conclusive results. Nevertheless, we presented the results of unadjusted odds ratios obtained with univariate logistic regression analysis in order to provide the potential predictors for the need for colectomy in patients with NEC.

Despite these limitations, to our knowledge, this is the first and largest study evaluating the clinical factors present in patients that underwent colectomy for neutropenic enterocolitis. We believe that our results could help general and colorectal surgeons when treating this selected morbid population.

\section{Conclusions}

In our study, patients were more likely to undergo colectomy if they developed organ failures and if they required ICU admission. Based on the severity of the disease and the high mortality rates after emergent colectomy, early surgical consultation and early surgery before the development of shock or organ failure should be pursued.

Authors' contributions Omar Vergara-Fernández and Mario Trejo-Avila designed this work, collected and interpreted the data, and drafted the manuscript. Omar Vergara-Fernández, Mario Trejo-Avila, Danilo Solórzano-Vicuña, Oscar Santes, and Noel Salgado-Nesme interpreted data, critically revised the manuscript, and performed overall supervision. All authors contributed to the final approval of the manuscript.

Funding information This research did not receive any specific grant from funding agencies in the public, commercial, or not-for-profit sectors.

\section{Compliance with ethical standards}

Conflict of interest The authors declare that they have no conflicts of interest.

Informed consent Informed consent was waived owing to the retrospective nature of the study.

\section{References}

1. Keng MK, Sekeres MA (2013) Febrile neutropenia in hematologic malignancies. Curr Hematol Malig Rep 8:370-378

2. Saillard C, Zafrani L, Darmon M, Bisbal M, Chow-Chine L, Sannini A, Brun JP, Ewald J, Turrini O, Faucher M, Azoulay E, Mokart D (2018) The prognostic impact of abdominal surgery in cancer patients with neutropenic enterocolitis: a systematic review and meta-analysis, on behalf the Groupe de Recherche en Réanimation Respiratoire du patient d'Onco-Hématologie (GRRR-OH). Ann Intensive Care 8:47

3. Sachak T, Arnold MA, Naini BV, Graham RP, Shah SS, Cruise M, Park JY, Clark L, Lamps L, Frankel WL, Theodoropoulos N, Arnold CA (2015) Neutropenic enterocolitis: new insights into a deadly entity. Am J Surg Pathol 39:1635-1642

4. Moir DH, Bale PM (1976) Necropsy findings in childhood leukaemia, emphasizing neutropenic enterocolitis and cerebral calcification. Pathology 8:247-258

5. Wagner ML, Rosenberg HS, Fernbach DJ, Singleton EB (1970) Typhlitis: a complication of leukemia in childhood. Am J Roentgenol 109:341-350

6. Song HK, Kreisel D, Canter R, Krupnick AS, Stadtmauer EA, Buzby G (1998) Changing presentation and management of neutropenic enterocolitis. Arch Surg 133:979-982

7. Gorschluter M, Mey U, Strehl J, Ziske C, Schepke M, SchmidtWolf IGH, Sauerbruch T, Glasmacher A (2005) Neutropenic enterocolitis in adults: systematic analysis of evidence quality. Eur J Haematol 75:1-13

8. Ullery BW, Pieracci FM, Rodney JR, Barie PS (2009) Neutropenic enterocolitis. Surg Infect 10:307-314

9. Hohenberger P, Buchheidt D (2005) Surgical interventions in patients with hematologic malignancies. Crit Rev Oncol Hematol 55: 83-91 
10. Portugal R, Nucci M (2017) Typhlitis (neutropenic enterocolitis) in patients with acute leukemia: a review. Expert Rev Hematol 10: 169-174

11. Freifeld AG, Bow EJ, Sepkowitz KA et al (2011) Clinical practice guideline for the use of antimicrobial agents in neutropenic patients with cancer: 2010 update by the Infectious Diseases Society of America. Clin Infect Dis 52:56-93

12. Uys A, Rapoport BL, Anderson R (2004) Febrile neutropenia: a prospective study to validate the multinational Association of Supportive Care of cancer (MASCC) risk-index score. Support Care Cancer 12:555-560

13. Charlson M, Szatrowski TP, Peterson J, Gold J (1994) Validation of a combined comorbidity index. J Clin Epidemiol 47:1245-1251

14. Singer M, Deutschman CS, Seymour CW, Shankar-Hari M, Annane D, Bauer M, Bellomo R, Bernard GR, Chiche JD, Coopersmith CM, Hotchkiss RS, Levy MM, Marshall JC, Martin GS, Opal SM, Rubenfeld GD, van der Poll T, Vincent JL, Angus DC (2016) The third international consensus definitions for sepsis and septic shock (Sepsis-3). JAMA 315:801-810

15. Baden LR, Bensinger W, Angarone M, Casper C, Dubberke ER, Freifeld AG, Garzon R, Greene JN, Greer JP, Ito JI, Karp JE, Kaul DR, King E, Mackler E, Marr KA, Montoya JG, Morris-Engemann A, Pappas PG, Rolston K, Segal B, Seo SK, Swaminathan S, Naganuma M, Shead DA (2012) National Comprehensive Cancer Network. Prevention and treatment of cancer-related infections. J Natl Compr Cancer Netw 10:1412-1445

16. Rodrigues FG, Da Silva G, Wexner SD (2017) Neutropenic enterocolitis. World J Gastroenterol 23:42-47

17. Cartoni C, Dragoni F, Micozzi A, Pescarmona E, Mecarocci S, Chirletti P, Petti MC, Meloni G, Mandelli F (2001) (2001) neutropenic enterocolitis in patients with acute leukemia: prognostic sig- nificance of bowel wall thickening detected by ultrasonography. J Clin Oncol 19:756-761

18. Smith TJ, Bohlke K, Armitage JO (2015) Recommendations for the use of white blood cell growth factors: American Society of Clinical Oncology clinical practice guideline update. J Oncol Pract 11:511513

19. Moir CR, Scudamore CH, Benny WB (1986) Typhlitis: selective surgical management. Am J Surg 151:563-566

20. Shamberger RC, Weinstein HJ, Delorey MJ, Levey RH (1986) The medical and surgical management of typhlitis in children with acute nonlymphocytic (myelogenous) leukemia. Cancer 57:603-609

21. Kunkle JM, Rosenthal D (1986) Management of the ileocecal syndrome. Dis Colon Rectum 29:196-199

22. Tokar B, Aydogdu S, Pasaoglu O, Ilhan H, Kasapoglu E (2003) Neutropenic enterocolitis: is it possible to break vicious circle between neutropenia and the bowel wall inflammation by surgery? Int J Color Dis 18:455-458

23. Williams N, Scott ADN (1997) Neutropenic colitis: a continuing surgical challenge. Br J Surg 84:1200-1205

24. Cunningham SC, Fakhry K, Bass BL, Napolitano LM (2005) Neutropenic enterocolitis in adults: case series and review of the literature. Dig Dis Sci 50:215-220

25. Koea AB, Shaw JHF (1989) Surgical management of neutropenic enterocolitis. Br J Surg 76:821-824

26. Alt B, Glass NR, Sollinger H (1985) Neutropenic enterocolitis in adults. Review of the literature and assessment of surgical intervention. Am J Surg 149:405-408

Publisher's note Springer Nature remains neutral with regard to jurisdictional claims in published maps and institutional affiliations. 